\title{
pH-Triggered Conformational Switching along the Membrane Insertion Pathway of the Diphtheria Toxin T-Domain
}

\author{
Alexey S. Ladokhin \\ Department of Biochemistry and Molecular Biology, The University of Kansas Medical Center, \\ Kansas City, KS 66160, USA; E-Mail: aladokhin@kumc.edu; Tel.: +1-913-588-0489; \\ Fax: +1-913-588-7440
}

Received: 8 July 2013; in revised form: 26 July 2013 / Accepted: 26 July 2013 /

Published: 6 August 2013

\begin{abstract}
The translocation (T)-domain plays a key role in the action of diphtheria toxin and is responsible for transferring the catalytic domain across the endosomal membrane into the cytosol in response to acidification. Deciphering the molecular mechanism of $\mathrm{pH}$-dependent refolding and membrane insertion of the T-domain, which is considered to be a paradigm for cell entry of other bacterial toxins, reveals general physicochemical principles underlying membrane protein assembly and signaling on membrane interfaces. Structure-function studies along the T-domain insertion pathway have been affected by the presence of multiple conformations at the same time, which hinders the application of high-resolution structural techniques. Here, we review recent progress in structural, functional and thermodynamic studies of the T-domain archived using a combination of site-selective fluorescence labeling with an array of spectroscopic techniques and computer simulations. We also discuss the principles of conformational switching along the insertion pathway revealed by studies of a series of T-domain mutants with substitutions of histidine residues.
\end{abstract}

Keywords: acid-induced conformational change; membrane protein insertion; histidine protonation; fluorescence; molecular dynamics; conformational switch

\section{Introduction}

Diphtheria toxin enters the cell via the endosomal pathway [1], which is shared by many other toxins, including botulinum, tetanus and anthrax [2-5]. The processes involved in the cellular entry 
of these toxins are complex and not fully understood. It is clear, however, that they have certain similarities with the entry pathway of diphtheria toxin: they involve receptor-mediated endocytosis followed by endosome acidification and $\mathrm{pH}$-triggered conformational change that results in membrane insertion of the transporting protein and the formation of a pore or a transient passageway through which the toxic enzymatic components enter the cell (Figure 1). In the case of diphtheria toxin, the bridging of the lipid bilayer is achieved through acid-induced refolding and membrane insertion of the translocation (T)-domain. Although T-domain has been a subject of numerous biophysical studies over the years [6-17], a consistent picture that would explain its action on a molecular level has yet to emerge. Here, we will review the results of structural and thermodynamic studies of T-domain refolding and membrane insertion obtained in our lab for the past decade.

Figure 1. Schematic representation of the endosomal pathway of cellular entry of diphtheria toxin, DT (adapted from [1]). The toxin consists of three domains: receptor-binding $(\mathrm{R})$ domain, responsible for initiating endocytosis by binding to the heparin-binding EGF (epidermal growth factor)-like receptor; translocation (T)-domain; and catalytic (C)-domain, blocking protein synthesis via modification of elongation factor 2. This review is concerned with $\mathrm{pH}$-triggered conformational change of the $\mathrm{T}$-domain resulting in refolding, membrane insertion and translocation of the C-domain (highlighted by the red rectangle).

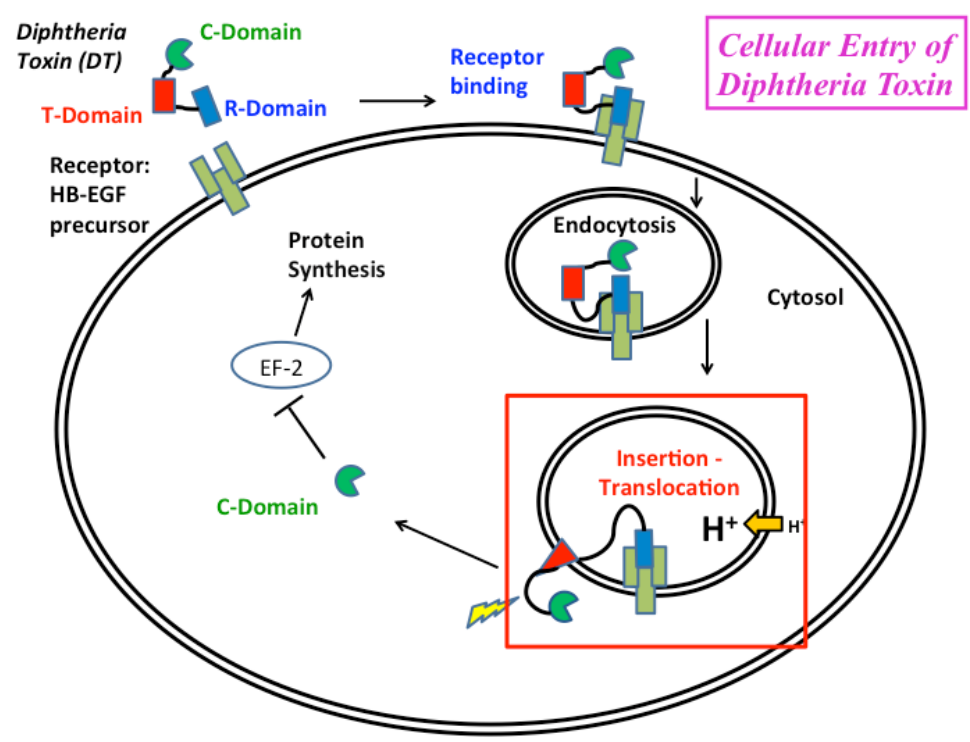

\section{Overview of the Insertion Pathway}

\subsection{Summary of Early Studies}

The crystallographic structure of diphtheria toxin T-domain in the water-soluble form $[18,19]$ (Figure 2A) provides a starting point for refolding/insertion studies. The protein consists of nine helices of various lengths (TH1-9), eight of which completely surround the most hydrophobic one, TH8. Helices 1 through 4 do not penetrate into the membrane, apparently, and are likely translocated along with the catalytic domain [20,21]. The two proposed models for the fully inserted functionally relevant state are the double dagger model [19] (derived from solution crystallographic structure) and 
the open-channel state model [9] (derived from numerous measurements of conductivity in planar bilayers [22-24]). Supporting evidence from other types of experiments is somewhat contradictory, and the flowing decade-old quote from the authors of the open-channel model still holds true: "by picking and choosing, one can select data from vesicle and cell membrane experiments supporting most of the T-domain topography" [9]. Part of the problem appears to be the difference in the nature of the information obtained by various methods and variations in sample preparation. Nevertheless, both conductivity measurements in planar bilayers [25] and spectroscopic measurements in vesicles [14] indicate that the active form of the T-domain is a monomer. In addition, a number of studies had reported the co-existence of multiple insertion intermediates [11-15,26]. While this conformational lability of the T-domain is not surprising, given the large-scale refolding required for insertion, it certainly complicates the application of high-resolution methods (e.g., X-ray crystallography and NMR) for structure determination of membrane-inserted T-domain. Our goal is to obtain atomistic representation of the T-domain structure along the entire insertion/translocation pathway into and across the lipid bilayer (illustrated by a scheme in Figure 3) and characterize the thermodynamics of the process. Below, we summarize our progress in achieving this task by combining various methods of fluorescence spectroscopy, such as fluorescence correlation spectroscopy, Förster resonance energy transfer and fluorescence lifetime quenching, and computer simulations.

Figure 2. (A) Backbone ribbon representation of the crystallographic structure of the T-domain [18]. Histidine 257 (red), critical for $\mathrm{pH}$-triggered refolding [27], is positioned between helices TH1-2 (yellow) and TH3-4 (blue). Other regions of the protein are: consensus membrane insertion domain, TH8-9, in brown and helices TH6-7 in grey. Two tryptophan residues are shown as space-filling models: W206 in yellow and W281 in grey. Lower panel (B) represents another view of the region surrounding H257, including H223 (purple), suggested to act as a safety latch preventing premature unfolding by modulating protonation of $\mathrm{H} 257$ [28].

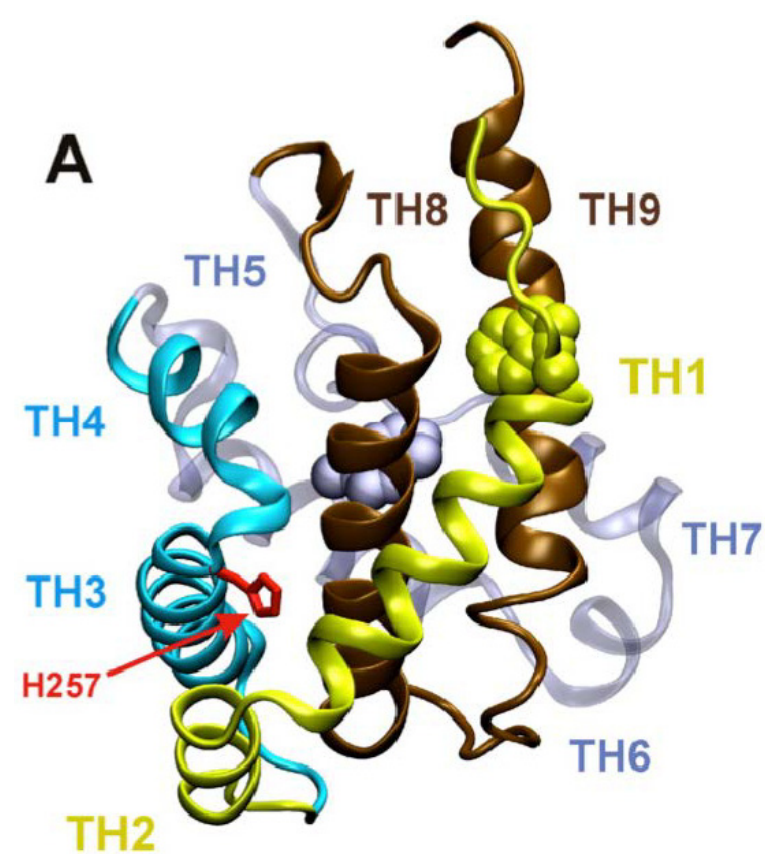

(A)

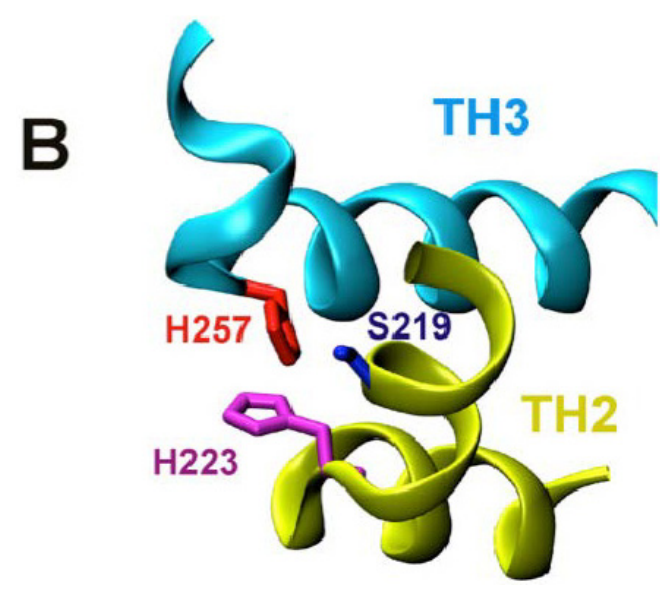

(B) 
Figure 3. Schematic representation of the $\mathrm{pH}$-dependent membrane insertion pathway of the diphtheria toxin T-domain (modified from [26]). Initial protonation, resulting in conversion of membrane-incompetent $\mathrm{W}$-state to membrane-competent $\mathrm{W}^{+}$-state, occurs primarily in the bulk of the solution. In the presence of membranes, this state rapidly associates with the bilayer to form an interfacial intermediate I-state. Subsequent insertion is facilitated by the presence of anionic lipids, which promote the formation of the insertion-competent $\mathrm{I}^{+}$-state and decrease the thermodynamic barrier for insertion into the TH8-9 helical hairpin. The two protonation steps responsible for the formation of conformations capable of membrane association ( $\mathrm{W}-$ to- $\mathrm{W}^{+}$transition, red rectangle) and insertion (I-to- $\mathrm{I}^{+}$transition, blue rectangle) have overlapping $\mathrm{pH}$ ranges, suggesting that additional protonation can occur at the same $\mathrm{pH}$ value, due to the shift of $\mathrm{pKa}$ values of titratable residues after their partitioning into the interfacial zone of the lipid bilayer. While the structure of the functional state of the T-domain on the membrane remains unknown, experimental evidence suggests coexistence of multiple transmembrane (TM)-inserted states, possibly affected by $\mathrm{pH}$ and membrane potential (see text and Figure 6 [29]).

\section{pH-Triggered Membrane Insertion-Translocation Pathway of Diphtheria Toxin T-Domain}

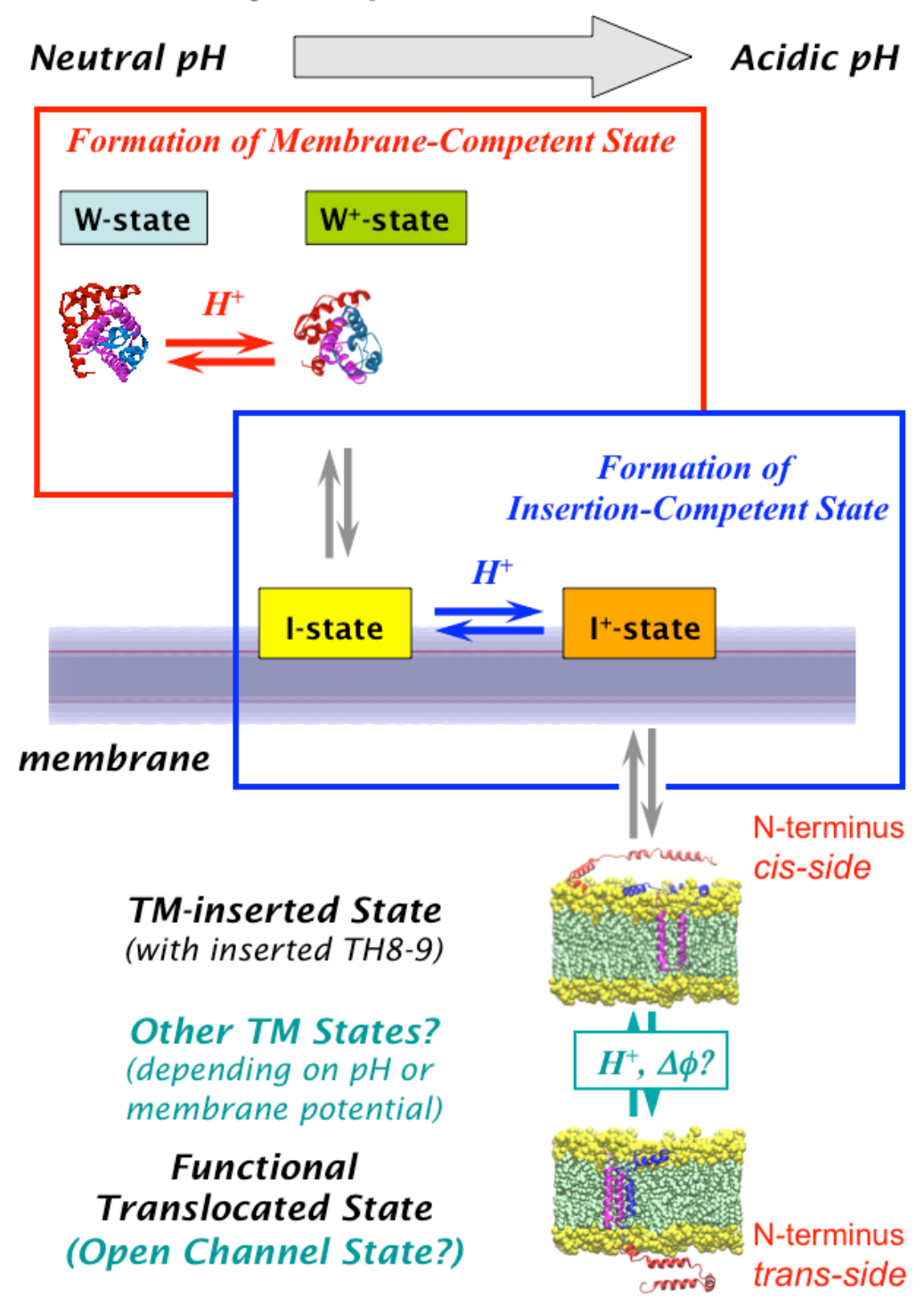




\section{2. pH-Dependent Formation of Membrane-Competent Form}

Formation of the membrane-competent form $\left(\mathrm{W}^{+}\right.$-state) of the T-domain is the first step along a complex pathway, leading from a soluble conformation with a known crystallographic structure (W-state), ultimately to membrane-inserted states, for which no high-resolution structural information is available. Initially, this state was identified through membrane binding at lipid saturation [26], and subsequently, its conformation has been characterized via a combination of spectroscopic experiments and all-atom Molecular Dynamics (MD) simulations [28]. pH-dependent transition between the $\mathrm{W}$-state and $\mathrm{W}^{+}$-state has a midpoint at $\mathrm{pH} 6.2$ (with a Hill coefficient, $n$, of two) and is over at pH 5.5 (Figure 4), i.e., in the $\mathrm{pH}$ range associated with early endosomes [30-32]. The structural rearrangements during formation of the $\mathrm{W}^{+}$-state are subtle, and this state was missed in early studies, which misidentified a molten globule state, formed at $\mathrm{pH}<5$, as a main membrane-binding species. Extensive microsecond-scale MD simulations performed with the ANTON supercomputer [33,34] reveal that the formation of the $\mathrm{W}^{+}$-state, triggered by the protonation of histidine residues, is not accompanied by the loss of structural compactness of the T-domain, while, nevertheless, resulting in substantial molecular rearrangements. A combination of simulation and experiments reveal the partial loss of secondary structure, due to unfolding of helices TH1 and TH2, and the loss of close contact between the $C$ - and $N$-terminal segments [28]. The structural changes accompanying the formation of the membrane-competent state ensure an easier exposure of the internal hydrophobic hairpin formed by helices TH8 and TH9, in preparation for its subsequent transmembrane insertion.

Figure 4. $\mathrm{pH}$-dependent conversion of the $\mathrm{T}$-domain from the soluble $\mathrm{W}$-state into the membrane-competent $\mathrm{W}^{+}$-state, identified through the following measurements of membrane binding at lipid saturation [26]: Fluorescence Correlation Spectroscopy-based mobility measurements (diamonds); measurements of FRET (Förster resonance energy transfer) between the donor-labeled T-domain and acceptor-labeled vesicles (circles). The solid line represents the global fit of the combined data [28].

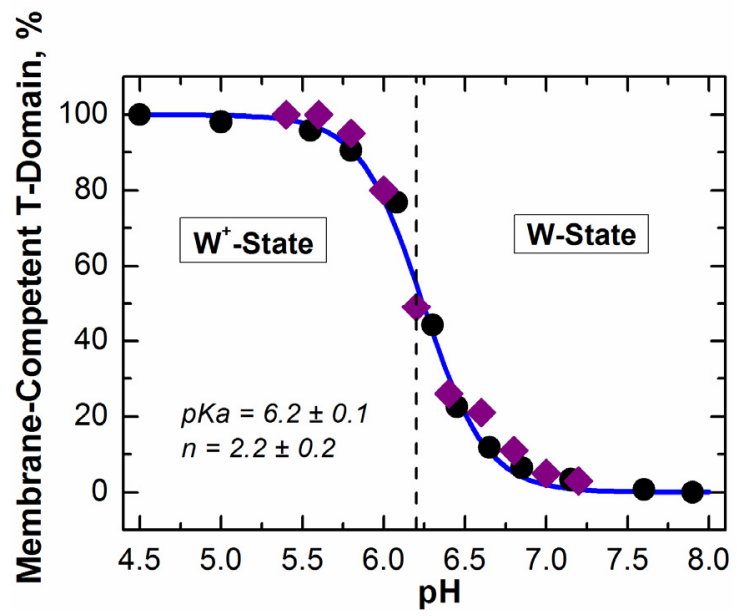

\subsection{Kinetic Insertion Intermediates}

Over the years, several research groups have presented compelling evidence for the T-domain adopting multiple conformations on the membrane [10-13,15], and yet, the kinetics of the transition 
between those forms has seldom been addressed. Several of those studies used intrinsic tryptophan fluorescence as a primary tool, which makes kinetic measurements difficult to implement and interpret, because of a low signal-to-noise ratio and a sometimes redundant spectroscopic response of tryptophan emission to binding, refolding and insertion. Previously, we have applied site-selective fluorescence labeling of the T-domain in conjunction with several specific spectroscopic approaches to separate the kinetics of binding (by FRET) and insertion (by environment-sensitive probe placed in the middle of TH9 helix) and explicitly demonstrate the existence of the interfacial insertion intermediate [26]. Direct observation of an interfacially refolded kinetic intermediate in the T-domain insertion pathway confirms the importance of understanding the various physicochemical phenomena (e.g., interfacial protonation [35], non-additivity of hydrophobic and electrostatic interactions [36,37] and partitioning-folding coupling $[38,39])$ that occur on membrane interfaces. This interfacial intermediate can be trapped on the membrane by the use of a low content of anionic lipids [26], which distinguishes theT-domain from other spontaneously inserting proteins, such as annexin B12, in which the interfacial intermediate is observed in membranes with a high anionic lipid content [40,41]. The latter can be explained by the stabilizing Coulombic interactions between anionic lipids and cationic residues present in the translocating segments of annexin. In contrast, in the T-domain, the only cationic residues in the TH8-9 segment are located in the top part of the helical hairpin (H322, H323, H372 and R377) and, thus, will not prevent its insertion. As a matter of fact, placing positive charges on the top of each helix is expected to assist insertion by providing interaction with anionic lipids. Indeed, triple replacement of $\mathrm{H} 322 / \mathrm{H} 323 / \mathrm{H} 372$ with either charged or neutral residues was observed to modulate the rate of insertion [42].

The reported non-exponential kinetics of insertion transition [26] clearly indicates the existence of at least a single intermediate populated after the initial binding event (formation of the I-state), but before the final insertion is achieved (formation of the T-state). Similarly to the membrane-competent state, we refer to this intermediate as an insertion-competent state. While the formation of the membrane-competent state (or membrane binding-competent state) leads to the conformation that can bind membrane, the formation of the insertion-competent state leads to the state that can adopt a TM conformation. The formation of this intermediate is both lipid- and $\mathrm{pH}$-dependent, with anionic lipids being essential for its formation (i.e., increasing the population of protein capable of insertion at a given $\mathrm{pH}$ ), as well as for increasing the overall insertion rate [26]. The mechanism for these effects is not known, although one can reasonably assume that variation in the local concentration of protons near membranes with different contents of anionic lipids can play a certain role. Other explanations involving direct interaction of anionic lipids with the intermediate and insertion-activated transient state should be considered, however.

\subsection{Insertion Pathway with Two Staggered pH-Dependent Transitions}

Various aspects of the $\mathrm{pH}$-triggered bilayer insertion of the $\mathrm{T}$-domain are illustrated using a pathway scheme in Figure 3. The initial protonation step, the formation of membrane-competent form $\mathrm{W}^{+}$, occurs in solution and depends little on the properties of the membrane [26]. (This is not always the case for $\mathrm{pH}$-triggered membrane protein insertion-for example, that of annexin B12, which inserts into a TM conformation at low $\mathrm{pH}$ in the absence of calcium. In the case of annexin, however, 
the formation of a membrane-competent state occurs not in the bulk of solution, but on the bilayer interface, and its $\mathrm{pH}$-dependence is modulated by lipid composition via surface potential [41]). The T-domain in this membrane-competent conformation is susceptible to aggregation, but it can be stabilized by fluorinated non-detergent surfactants that act as insertion chaperones [14,43]. Application of such surfactants is essential for equilibrium thermodynamic studies of insertion [17], but is not practical for kinetic studies. In the presence of membranes, the $\mathrm{W}^{+}$-state rapidly associates with the bilayer interface (I-state). It is not clear what structural rearrangements are associated with this transition. Final TM insertion requires the formation of the insertion-competent form $\left(\mathrm{I}^{+}\right)$, which is populated in another $\mathrm{pH}$-dependent transition and depends strongly on the fraction of anionic lipids and less on the nature of lipid headgroups [26,29].

An important aspect of the insertion pathway is that the two $\mathrm{pH}$-dependent transitions, $\mathrm{W}$-to- $\mathrm{W}^{+}$ and I-to- $\mathrm{I}^{+}$, are not sequential, but staggered, i.e., the second transition starts well before the first one is completed [26] (compare Figures 4 and 5). This implies additional protonation of the T-domain at the same $\mathrm{pH}$ to the membrane interface, which can be explained by the change in the pKa of titratable groups responsible for insertion once they are removed from an aqueous environment. The acidic residues, E349, D352 and E362, located in the TH8-9 insertion hairpin, are the likely candidates. Moreover, it is possible that their protonation will be affected by the presence of negative charges on the membrane, which would explain the promotion of insertion by anionic lipids. Quite possibly, the existence of overlapping protonation transitions is an essential feature of all $\mathrm{pH}$-driven membrane protein interactions.

Figure 5. pH-dependent transmembrane (TM) insertion of the T-domain into the vesicles with various lipid compositions measured by fluorescence of the environment-sensitive probe, NBD ( $N$-(7-nitro-2-1,3-benzoxadiazol-4-yl), attached to a single cysteine in the middle of TH9 helix [26]. Insertion is promoted by anionic lipids (molar ratios of POPC(palmitoyloleoylphosphatidylcholine)-to-POPG(palmitoyloleoylphosphatidylglycerol) three-to-one1 shown in red and one-to-three in blue). No TM insertion is observed when the POPC-to-POPG ratio is nine-to-one (green); even the protein is completely bound to the membrane in the interfacial I-state (Figure 3). This lipid-dependent TM insertion is independently confirmed by topology experiments [26] based on the fluorescence lifetime quenching method [44].

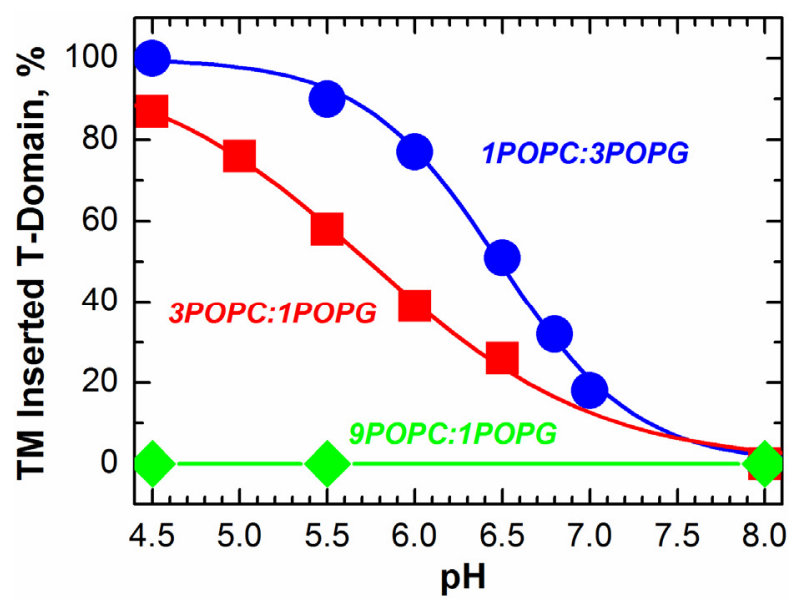




\subsection{Multitude of TM-Inserted States Conundrum}

One of the possible reasons for the absence of a high-resolution structure of the T-domain in the final inserted conformation could be the fact that there is no single conformation in the transmembrane state, but, rather, a collection of states with different folds and topologies. It is clear that one can hardly expect the T-domain to form a regular large pore (for example, one similar to that of anthrax toxin [5]), and it is possible that the molecular species responsible for the physiological function of catalytic domain translocation is formed only transiently. Nevertheless, certain general features of the family of inserted states can be identified. For example, most studies agree that in the inserted state (or states), a hydrophobic helical hairpin, TH8-9, adopts a TM conformation [6,10,26]. The insertion of this consensus domain, however, appears to depend on the exact nature of the sample. The EPR measurements that indicate a TM conformation of these helices [6] are performed using large unilamellar vesicles (LUV) as a membrane system and using a lipid-to-protein ratio of $R_{\mathrm{i}}=500$. Normally, the inserted T-domain is separated from the rest of the sample by centrifugation prior to Electron Paramagnetic Resonance measurements. On the other hand, it has been suggested that efficient insertion requires either a high protein concentration (or low $R_{\mathrm{i}}, \sim 400$ ) or the use of short-chained lipids, such as dimyristoylphosphatidylcholine [10], and can proceed only in small unilamellar vesicles (SUV) [10], but not in LUV [11]. (Unlike larger extruded LUV, sonicated SUV are not equilibrium structures and can result in irregular protein and peptide penetration, as discussed in [45]). In contrast, we were able to utilize the fluorescence lifetime quenching topology method [44] to demonstrate that TH8-9 does adopt a TM conformation in LUV composed of POPC:POPG mixtures, even at $R_{\mathrm{i}}=3,000$, but in a lipid-dependent manner, with anionic lipids greatly favoring the insertion [26]. (It is possible that the low content of anionic lipids in the sample is responsible for the reported conformation of the T-domain with helices parallel to the interface [46]). In addition, our mutagenesis data, discussed in detail below, indicate that insertion of TH8-9 is not necessarily followed by proper insertion of the rest of the protein or translocation of the terminus [42]. It is clear that identifying and characterizing membrane-inserted states constitutes a bottleneck in deciphering the mechanism of action of the T-domain and that progress in this area will require application of new methods and approaches. One of the promising directions of such studies appears to be a utilization of integrated methodologies, combining various spectroscopic techniques with computer simulations.

\section{Role of Histidine Protonation in Conformational Switching}

\subsection{Mutagenesis Studies}

Two groups of residues are expected to undergo protonation in the range of $\mathrm{pH}$ relevant to physiological changes inside the endosome: acidic residues (aspartic and glutamic acid), which will lose negative charge upon acidification, and histidines, which will gain a positive charge. Histidine protonation has been implicated in the biological activity of other toxins, including anthrax [47] and aerolysin [48]. It has been suggested that the protonation of the six native histidines of the T-domain makes a favorable thermodynamic contribution to the formation of the interfacial intermediate state of the T-domain [13] and is implicated in the modulation of insertion by anionic lipids [26]. The role of histidines in the action of T-domain has been addressed by Perier et al. [16], who studied the 
membrane interactions of a series of mutants with H-to-F replacements. Such a replacement results in the potential introduction of strong, non-native hydrophobic interactions with the lipid bilayer [49]. In our studies, we have designed an alternative mutagenesis strategy, which is based on comparison of the biophysical and physiological properties of the T-domain, wild type (WT), with those of (a) mutants with neutral, but not hydrophobic residues (H-to-Q replacement) and (b) those with $\mathrm{pH}$-independent positive charge (H-to-R or H-to-K replacements) [27,29,42].

\subsubsection{Role of $\mathrm{H} 257$ as a Major Component of pH-Dependent Conformational Switch}

The effects of systematic replacement (one-by-one and in groups) of all six native histidines of the T-domain with either glutamine or arginine residues on folding in solution was studied by means of circular dichroism (CD) and intrinsic fluorescence [27]. Some replacements (e.g., those of H251) caused pronounced misfolding, while others had only moderate effect on changes of secondary structure. The most intriguing result was obtained with substitutions of $\mathrm{H} 257$ : a replacement with the neutral glutamine caused little effect at neutral $\mathrm{pH}$, while replacement with the charged arginine caused substantial unfolding. Remarkably, this unfolding was completely reversed by membrane insertion at acidic $\mathrm{pH}$, where $\mathrm{CD}$ and fluorescence spectra of H257R mutant regained a WT-like appearance. This behavior is reminiscent of that of intrinsically disordered proteins, with the lipid bilayer playing the role of a ligand, causing gain of structure. Interesting results were also revealed by studies of permeabilization of vesicles loaded with the fluorophore/quencher pair by H257R and H257Q mutants of the T-domain [27]. Whereas both mutants exhibit similar final levels of permeabilization at $\mathrm{pH} 4.5$, the kinetics of release caused by the H257Q mutant is orders of magnitude slower than that of H257R or WT. This indicates that removing the positive charge on $\mathrm{H} 257$ significantly affects $\mathrm{pH}$-triggered conformational switching in the T-domain, but does not eliminate it completely, suggesting that such switching is redundant (i.e., it can be triggered by multiple residues). Consistent with this mechanism, introducing a $\mathrm{pH}$-independent positive charge at this position is expected to result in an increased activity at neutral $\mathrm{pH}$, which is, indeed, observed for the H257R mutant [27].

The central role of protonation of $\mathrm{H} 257$ in destabilizing the folded structure of the T-domain in solution has been confirmed with thermodynamic integration calculations based on a series of MD simulations. The energy penalty for protonation of $\mathrm{H} 257$ in the context of the $\mathrm{W}$-state was found to be $6.9 \mathrm{kcal} / \mathrm{mole}(10.2 \mathrm{kcal} / \mathrm{mole}$, if easily protonatable $\mathrm{H} 223$ is already charged), which is the highest among the six histidines [28]. This penalty alone is quite sufficient to overcome the folding free energy of the T-domain, which is on the order of 6-7 kcal/mole. We will further discuss the implications of theoretical predictions of protonation of $\mathrm{H} 223$ and $\mathrm{H} 257$ based on Poisson-Boltzmann calculations of pKa distributions in the subsequent section.

\subsubsection{Role of C-Terminal Histidine Cluster in Membrane Insertion and Translocation}

C-terminal histidine residues, $\mathrm{H} 322, \mathrm{H} 323$, and $\mathrm{H} 372$, have a peculiar location, flanking the consensus insertion domain, TH8-9. The replacement of the three $C$-terminal histidine residues in triple-R or triple-Q mutants prevents effective translocation of the $\mathrm{N}$-terminus, while introduction of these mutations in the full-length toxin results in the decrease of its potency [42]. In the context of isolated T-domain, these mutations cause loss of characteristic conductance in planar bilayers. 
Surprisingly, these mutations do not affect general folding in solution, protein interaction with the membranes and insertion of the consensus transmembrane helical hairpin, TH8-9 [42]. This indicates the existence of multiple inserted states of the T-domain with various membrane topologies (Figure 3, lower panel). Thus, the C-terminal histidine residues are critical for the transition from the inserted intermediate state to the open-channel state in the insertion/translocation pathway of the T-domain. Recently, we have demonstrated that these effects are mainly due to the replacement of H322, although other histidines also influence the insertion pathway [29].

Figure 6. Role of C-terminal histidines in modulating membrane-insertion pathway of the T-domain [29,42]. (A) C-terminal histidines, H322, H323 and H372, are located on top of the insertion unit comprising a helical hairpin TH8-9 (highlighted in brown) in the crystal structure of the soluble form of the diphtheria toxin T-domain. Tryptophan residues W206 and W281 are shown in yellow, and the rest of the protein is shown in grey; (B) Schematic representation of the differences in the insertion process of the WT T-domain and its mutants. Top (WT T-domain): upon initial destabilization of the WT T-domain and its association with the lipid bilayer, the $N$-terminal region of the protein adopts a conformation that leads to the insertion of the TH8-9 unit into the bilayer. The $N$-terminal region refolds to form the open channel state (OCS). Bottom (mutants with $C$-terminal histidine replacements): membrane interaction of these mutants results in a different conformation from that of the WT, specifically in the more exposed $N$-terminal part, as revealed by a red-shifted fluorescence. While the initial insertion of TH8-9 is not compromised by the mutations [42], the replacement of $C$-terminal histidines, especially that of H322, affects efficient folding of the T-domain into the OCS [29].
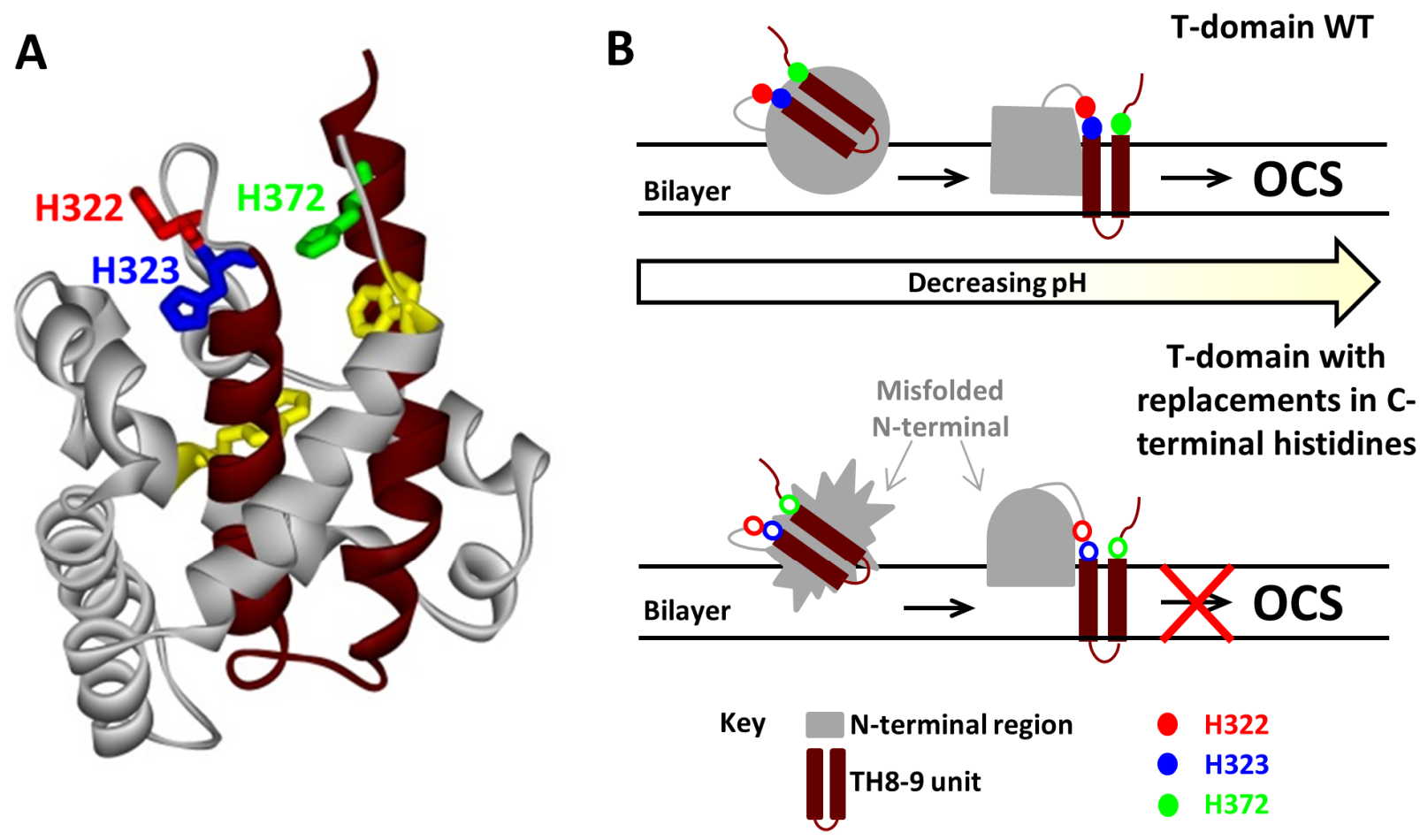

We illustrate the role of $C$-terminal histidines in the scheme summarizing membrane insertion of the WT T-domain and the mutants carrying substitutions of the $C$-terminal histidines (Figure 6). Upon 
initial formation of the membrane-competent state and binding to the membrane, the process continues through the insertion of TH8-9 into the bilayer and the subsequent refolding of the rest of the protein, until reaching the open-channel state [26]. It is proposed that the $C$-terminal histidines are involved in guiding the conformation of the $N$-terminal region through productive folding intermediate states towards the Open Channel State (OCS). There is no high-resolution structure of the OCS available (or that of any membrane-associated intermediate); however, the electrophysiological data are consistent with helices TH8, TH9 and TH5 adopting a transmembrane conformation [9]. When $C$-terminal histidines are replaced, the protein still undergoes a proper $\mathrm{pH}$-dependent destabilization in solution, binds to membranes [29] and inserts a TH8-9 helical hairpin [42] similar to that of the WT. Histidine replacement, however, leads to the formation of a non-productive intermediate that is detected by spectral measurements of intrinsic fluorescence, indicating greater exposure of W206 and W281 to the aqueous phase at $\mathrm{pH}$ values of $\sim 6-6.5$. The replacement of H322 appears to be particularly damaging, as the corresponding mutants tend to misfold and, possibly, aggregate on the membrane, dramatically reducing the number of properly folded and functional channels. Interestingly, the replacement of $\mathrm{H} 322$ with the charged or neutral residue has a similar effect on the folding pathway, which is different from replacements of another critical residue, H257, involved in destabilization of the folded structure in solution [27] discussed above.

\subsection{Computer Simulation Studies}

Cellular entry of DT starts with receptor-mediated endocytosis [1], but the critical step occurs inside the endosome, resulting in bridging the membrane of the compartment by the T-domain, followed by translocation of the catalytic domain. How do the above-discussed biophysical studies performed in vitro or in silico relate to the process of cellular entry, and what can we learn from them about molecular mechanism of in vivo action of the T-domain? The initial states on the insertion pathway (Figure 3) can be a map of cellular entry (Figure 1) in the following way: the membrane-incompetent $\mathrm{W}$-state corresponds to the state outside the cell, while the protonated membrane-competent $\mathrm{W}^{+}$-state corresponds to the state inside the endosome. The $\mathrm{pH}$ range of 5.5-6.5 measured for the $\mathrm{W}$-to- $\mathrm{W}^{+}$ in vitro (Figure 4) corresponds well to the $\mathrm{pH}$ range in early endosomes [30-32]. Biophysical experiments and MD simulations allow us to take a look at how the T-domain prepares to make cellular entry with molecular resolution. Recent results demonstrate with atomistic detail how protonation of histidines triggers a conformational change that prepares the T-domain for membrane binding and insertion (e.g., breakage of long TH-1 helix and exposure of the TH8-9 consensus insertion domain) [28]. In addition to these structural rearrangements, our calculations reveal important thermodynamic implications of histidine protonation for modulating cellular action of the T-domain. We illustrate these findings in Figure 7, which presents the results of Poisson-Boltzmann calculation of $\mathrm{pKa}$ values for all six histidines of the diphtheria toxin T-domain, both in $\mathrm{W}$ - and $\mathrm{W}^{+}$-states.

The advantage of long microsecond-scale MD simulations is that they allow one to explore in great detail the distribution of conformational states and characterize their thermodynamic properties, such as the pKas of titratable groups. As a result, rather than analyzing a single average pKa available for static crystallographic structure, we have at our disposal entire distributions (Figure 7). It is remarkable that the only two histidine residues to exhibit a double-headed distribution of pKas, namely $\mathrm{H} 257$ 
and H322 [28], are those that were identified through mutagenesis as being critical for refolding in solution [27] and on membrane interface [29]. We hypothesize that the bimodal distribution of pKas is a hallmark of residues involved in $\mathrm{pH}$-triggered conformational switching, as it allows it to become protonated via a high-pKa mode, but perturbs the structure via a low-pKa mode.

Figure 7. pKa distributions for $N$-terminal (a,c) and $C$-terminal (b,d) histidine residues of the T-domain calculated in Poisson-Boltzmann approximation from Molecular Dynamics (MD) traces for the membrane-incompetent $\mathrm{W}$-state $(\mathrm{a}, \mathrm{b})$ and the membrane-competent $\mathrm{W}^{+}$-state (c,d) (data for the entire MD trace are published in [28]). Remarkably, the only two residues with bimodal distribution of $\mathrm{pKa}$ are those that were shown to be critical to refolding in solution (H257) and to guiding the insertion from the membrane interface (H322) by mutagenesis studies [27,29]. Note that under conditions of endosomal $\mathrm{pH}$, all six histidines are predicted to be protonated in the $\mathrm{W}^{+}$-state. Coupling of histidine protonation to the conformational change results in a complete conversion of the T-domain to the membrane-competent state by $\mathrm{pH} 5.5$, which is observed experimentally (Figure 4).
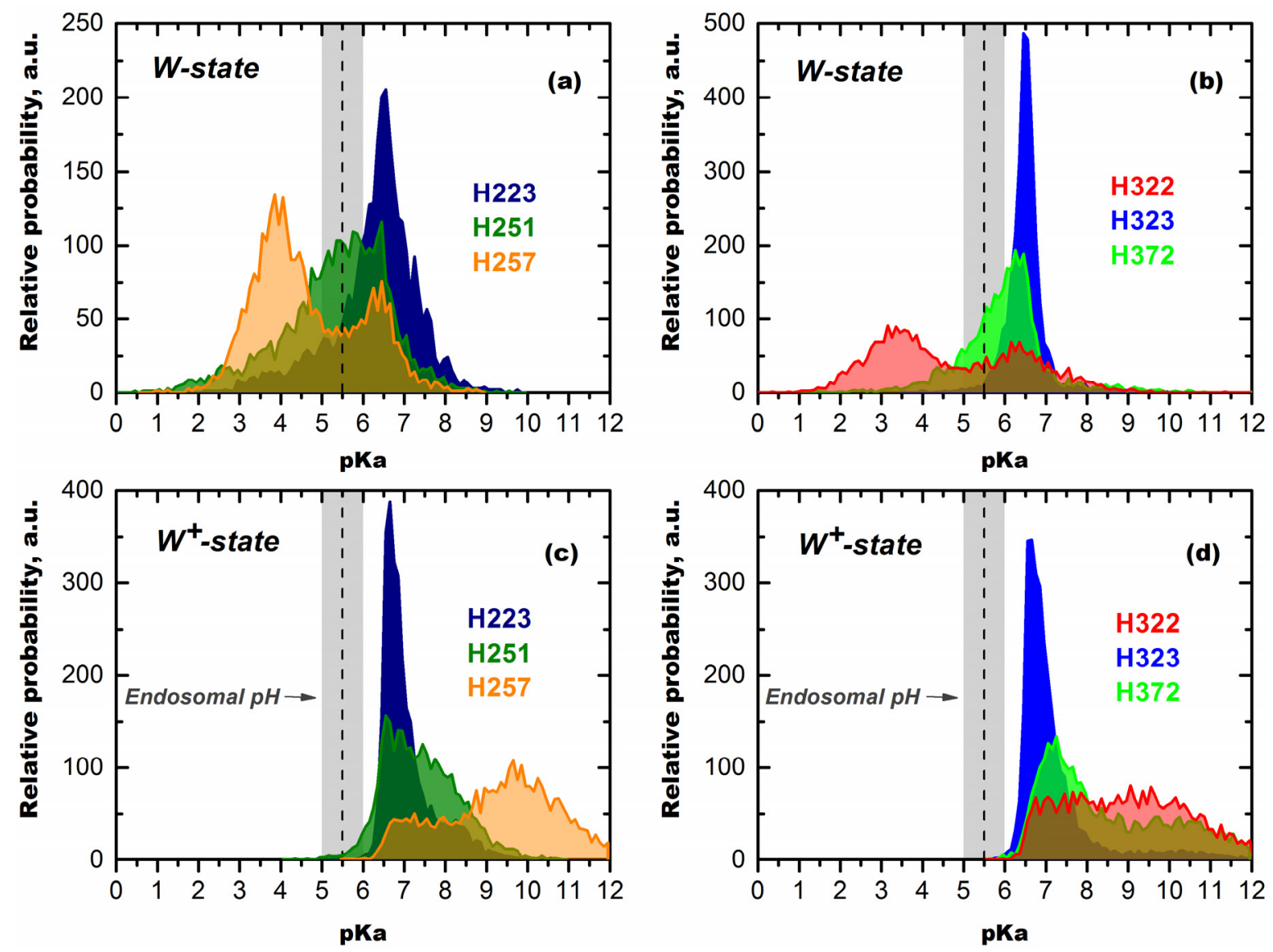

Mechanisms of $\mathrm{pH}$-Trigger and Safety Latch Suggested by MD Simulations

In the $\mathrm{W}$-state, $\mathrm{H} 223$ exhibits a unimodal $\mathrm{pKa}$ distribution with a maximum at $\mathrm{pKa} 6.5$ (Figure 7a), which makes it susceptible to protonation, even before endosomal encapsulation of the T-domain. In contrast, $\mathrm{H} 257$ has a much lower average $\mathrm{pKa}$ of 4.7, distributed at two peaks centered at $\mathrm{pH} 4$ and 6.3. The distribution for $\mathrm{H} 257$ is shifted even further to acidic $\mathrm{pH}$ by approximately 1.5 units, when 
calculation is repeated, assuming H223 is already protonated (not shown). These differences are consistent with the thermodynamic integration calculations discussed above, which suggest that protonation of $\mathrm{H} 257$ in the context of the $\mathrm{W}$-state is very costly energetically, especially when $\mathrm{H} 223$ is protonated [28]. This does not mean, however, that one needs to reach a $\mathrm{pH}$ below four to protonate $\mathrm{H} 257$ in the cell, because the T-domain undergoes a protonation-coupled refolding to the $\mathrm{W}^{+}$-state. To illustrate the concept of this linked equilibrium, consider a particular T-domain molecule that adopts a conformation with a pKa of 5.5 for $\mathrm{H} 257$ and, thus, has a 50\% probability of a proton on this histidine at $\mathrm{pH}$ 5.5. The molecule can remain in this conformation and, eventually, lose a proton or go to another conformation in the $\mathrm{W}$-state ensemble without major structural rearrangements. Alternatively, it can undergo a proton-triggered conformational change leading to the formation of a membrane-competent $\mathrm{W}^{+}$-state, in which the probability of remaining protonated at $\mathrm{pH} 5.5$ is $100 \%$ (Figure 7). In accordance with the Le Chatelier principle, this coupling will result in complete transfer of the entire population toward the protonated $\mathrm{W}^{+}$-state at $\mathrm{pH} 5.5$.

The $\mathrm{pKa}$ distributions calculated for the membrane-competent state (Figure $7 \mathrm{c}, \mathrm{d}$ ) indicate that all histidine residues will remain protonated in the endosome. As described above, the implications of this coupling of protonation and conformational change are such that, even at $\mathrm{pH} 5.5$, all molecules of the T-domain will undergo a transition to the $\mathrm{W}^{+}$-state, which is indeed observed experimentally (Figure 4). According to the pKa calculations, this transition would have started before the endosomal internalization, if it were not for the effects of H223 lowering the pKa distribution of H257 [28]. Therefore, we suggest that easily protonatable H223 acts as a safety latch for preventing the triggering of the conformational change in the T-domain prematurely. The premature refolding of the T-domain outside the endosomal compartment would be non-productive for the following reasons: (1) the $\mathrm{pH}$ is not right for the subsequent states of the insertion pathway and (2) the catalytic domain has not yet undergone an acid-induced destabilization and is not ready to be translocated into the cytosol. We hypothesize that this may be important physiologically, because, otherwise, the protonation of $\mathrm{H} 257$ would have caused substantial unfolding before the endosomal compartment is reached and would trigger a non-productive interaction with the membrane at an early stage of the insertion pathway. Thus, H223 can be compared to a safety device, which reduces protonation of the crucial H257 by further shifting its $\mathrm{pKa}$ and holding it in a state resembling a loaded spring, until the protein is poised for translocation in the endosomal compartment. Once acidification of an endosome lowers $\mathrm{pH}$ sufficiently for the protonation of $\mathrm{H} 257$ to occur, the safety latch can no longer hold, and the spring is released, causing the conformational change that results in formation of the membrane-competent state, membrane insertion and translocation.

\section{Perspectives and Applications}

The Diphtheria toxin T-domain has been shown to implement its function-translocation of the catalytic domain across the endosomal membrane under acidic conditions - by itself, without the help of any additional protein component [20]. It has also been suggested that it assists other partially unfolded proteins across the lipid bilayer [50], indicating a general, rather than specific translocation pathway. Recently, this membrane-translocating ability of the T-domain has been utilized to improve cellular delivery of poly(ethylenimine)-based vectors during gene transfection [51]. Diphtheria toxin 
has been utilized as a prospective anti-cancer agent for the targeted delivery of cytotoxic therapy to cancer cells [52-65]. Normally, the targeting is achieved by deleting the cell receptor-binding $\mathrm{R}$-domain and combining the remaining portion (containing $\mathrm{T}$ - and $\mathrm{C}$-domains) with proteins that selectively bind to the surface of cancer cells (one such fusion protein, which contains human interleukin-2 and truncated diphtheria toxin, is approved for use in cutaneous T-cell lymphoma $[54,59,60]$ ). While it has been assumed that "receptorless" toxin cannot bind to and kill cells, a recent study demonstrated that recombinant DT385 with a deleted R-domain is, in fact, cytotoxic to a variety of cancer cell lines [52]. Because cancerous cells are known to produce a slightly acidic environment, it is likely that the targeting of "receptorless" toxin is assured by $\mathrm{pH}$-triggered membrane insertion of the T-domain in a fashion similar to that of the pHLIP peptide [66,67]. Understanding the molecular mechanism of T-domain action will influence our ability to rationally design drug delivery systems based on $\mathrm{pH}$-dependent conformational switching.

Biophysical studies of the $\mathrm{pH}$-triggered action of the diphtheria toxin T-domain are expected to impact not only the field of cellular entry of toxins or targeted cellular delivery of therapy, but would also advance our understanding of general physicochemical principles underlying conformational switching in proteins. For example, a number of proteins from the Bcl-2 family, carrying out both pro-apoptotic and anti-apoptotic functions, have been demonstrated to have a solution fold dominated by a hairpin composed of long hydrophobic helices similar to those of the diphtheria toxin T-domain [68,69]. Additionally, similar to the T-domain, they have been shown to form ion channels in artificial bilayers [70]. Although it is not clear exactly how these proteins modulate the apoptotic response, a change in membrane topology has been suggested to play a role [71]. The models proposed for their membrane insertion are almost exclusively based on data generated for membrane insertion of the T-domain. Notably, these models have not been tested experimentally and are based on structural similarities of the solution fold, rather than any thermodynamic analysis of membrane-binding propensities. Deciphering the physicochemical rules governing interactions of the diphtheria toxin T-domain with membranes of various lipid compositions will help generate testable hypotheses of the mode of interaction of the $\mathrm{Bcl}-2$ proteins with the outer mitochondrial membrane during apoptosis.

\section{Acknowledgments}

The author is grateful to the following members of his lab for their contribution to this project and help in preparation of illustrations: Mauricio Vargas-Uribe, Alexander Kyrychenko and Mykola V. Rodnin. The research from our lab described in this review has been supported by NIH GM069783.

\section{Conflict of Interest}

The author declares no conflict of interest.

\section{References}

1. Murphy, J.R. Mechanism of diphtheria toxin catalytic domain delivery to the eukaryotic cell cytosol and the cellular factors that directly participate in the process. Toxins 2011, 3, 294-308. 
2. Hoch, D.H.; Romero-Mira, M.; Ehrlich, B.E.; Finkelstein, A.; DasGupta, B.R.; Simpson, L.L. Channels formed by botulinum, tetanus, and diphtheria toxins in planar lipid bilayers: Relevance to translocation of proteins. Proc. Natl. Acad. Sci. USA 1985, 82, 1692-1696.

3. Neale, E.A. Moving across membranes. Nat. Struct. Biol. 2003, 10, $2-3$.

4. Koriazova, L.K.; Montal, M. Translocation of botulinum neurotoxin light chain protease through the heavy chain channel. Nat. Struct. Biol. 2003, 10, 13-18.

5. Collier, R.J.; Young, J.A. Anthrax toxin. Annu. Rev. Cell Dev. Biol. 2003, 19, 45-70.

6. Oh, K.J.; Zhan, H.; Cui, C.; Hideg, K.; Collier, R.J.; Hubbell, W.L. Organization of diphtheria toxin T domain in bilayers: A site-directed spin labeling study. Science 1996, 273, 810-812.

7. Oh, K.J.; Zhan, H.; Cui, C.; Altenbach, C.; Hubbell, W.L.; Collier, R.J. Conformation of the diphtheria toxin $\mathrm{t}$ domain in membranes: A site-directed spin-labeling study of the TH8 helix and TL5 loop. Biochemistry 1999, 38, 10336-10343.

8. Kachel, K.; Ren, J.H.; Collier, R.J.; London, E. Identifying transmembrane states and defining the membrane insertion boundaries of hydrophobic helices in membrane-inserted diphtheria toxin $\mathrm{T}$ domain. J. Biol. Chem. 1998, 273, 22950-22956.

9. Senzel, L.; Gordon, M.; Blaustein, R.O.; Oh, K.J.; Collier, R.J.; Finkelstein, A. Topography of diphtheria toxin's T domain in the open channel state. J. Gen. Physiol. 2000, 115, 421-434.

10. Zhao, G.; London, E. Behavior of diphtheria toxin $t$ domain containing substitutions that block normal membrane insertion at Pro345 and Leu307: Control of deep membrane insertion and coupling between deep insertion of hydrophobic subdomains. Biochemistry 2005, 44, 4488-4498.

11. Wang, Y.; Malenbaum, S.E.; Kachel, K.; Zhan, H.J.; Collier, R.J.; London, E. Identification of shallow and deep membrane-penetrating forms of diphtheria toxin $\mathrm{T}$ domain that are regulated by protein concentration and bilayer width. J. Biol. Chem. 1997, 272, 25091-25098.

12. Chenal, A.; Savarin, P.; Nizard, P.; Guillain, F.; Gillet, D.; Forge, V. Membrane protein insertion regulated by bringing electrostatic and hydrophobic interactions into play. A case study with the translocation domain of the diphtheria toxin. J. Biol. Chem. 2002, 277, 43425-43432.

13. Ladokhin, A.S.; Legmann, R.; Collier, R.J.; White, S.H. Reversible refolding of the diphtheria toxin T-domain on lipid membranes. Biochemistry 2004, 43, 7451-7458.

14. Palchevskyy, S.S.; Posokhov, Y.O.; Olivier, B.; Popot, J.L.; Pucci, B.; Ladokhin, A.S. Chaperoning of insertion of membrane proteins into lipid bilayers by hemifluorinated surfactants: Application to diphtheria toxin. Biochemistry 2006, 45, 2629-2635.

15. Montagner, C.; Perier, A.; Pichard, S.; Vernier, G.; Menez, A.; Gillet, D.; Forge, V.; Chenal, A. Behavior of the $N$-terminal helices of the diphtheria toxin T domain during the successive steps of membrane interaction. Biochemistry 2007, 46, 1878-1887.

16. Perier, A.; Chassaing, A.; Raffestin, S.; Pichard, S.; Masella, M.; Menez, A.; Forge, V.; Chenal, A.; Gillet, D. Concerted protonation of key histidines triggers membrane interaction of the diphtheria toxin T domain. J. Biol. Chem. 2007, 282, 24239-24245.

17. Posokhov, Y.O.; Rodnin, M.V.; Das, S.K.; Pucci, B.; Ladokhin, A.S. FCS study of the thermodynamics of membrane protein insertion into the lipid bilayer chaperoned by fluorinated surfactants. Biophys. J. 2008, 95, L54-L56.

18. Bennett, M.J.; Eisenberg, D. Refined structure of monomeric diphtheria toxin at 2.3 å resolution. Protein Sci. 1994, 3, 1464-1475. 
19. Weiss, M.S.; Blanke, S.R.; Collier, R.J.; Eisenberg, D. Structure of the isolated catalytic domain of diphtheria toxin. Biochemistry 1995, 34, 773-781.

20. Oh, K.J.; Senzel, L.; Collier, R.J.; Finkelstein, A. Translocation of the catalytic domain of diphtheria toxin across planar phospholipid bilayers by its own T domain. Proc. Natl. Acad. Sci. USA 1999, 96, 8467-8470.

21. Senzel, L.; Huynh, P.D.; Jakes, K.S.; Collier, R.J.; Finkelstein, A. The diphtheria toxin channel-forming $\mathrm{T}$ domain translocates its own $\mathrm{NH}_{2}$-terminal region across planar bilayers. J. Gen. Physiol. 1998, 112, 317-324.

22. Mindell, J.A.; Silverman, J.A.; Collier, R.J.; Finkelstein, A. Structure function relationships in diphtheria toxin channels. II. A residue responsible for the channel's dependence on trans $\mathrm{pH}$. J. Membr. Biol. 1994, 137, 29-44.

23. Mindell, J.A.; Silverman, J.A.; Collier, R.J.; Finkelstein, A. Structure-function relationships in diphtheria toxin channels. III. Residues which affect the cis $\mathrm{pH}$ dependence of channel conductance. J. Membr. Biol. 1994, 137, 45-57.

24. Huynh, P.D.; Cui, C.; Zhan, H.J.; Oh, K.J.; Collier, R.J.; Finkelstein, A. Probing the structure of the diphtheria toxin channel: Reactivity in planar lipid bilayer membranes of cysteine-substituted mutant channels with methanethiosulfonate derivatives. J. Gen. Physiol. 1997, 110, 229-242.

25. Gordon, M.; Finkelstein, A. The number of subunits comprising the channel formed by the T domain of diphtheria toxin. J. Gen. Physiol. 2001, 118, 471-480.

26. Kyrychenko, A.; Posokhov, Y.O.; Rodnin, M.V.; Ladokhin, A.S. Kinetic intermediate reveals staggered $\mathrm{pH}$-dependent transitions along the membrane insertion pathway of the diphtheria toxin t-domain. Biochemistry 2009, 48, 7584-7594.

27. Rodnin, M.V.; Kyrychenko, A.; Kienker, P.; Sharma, O.; Posokhov, Y.O.; Collier, R.J.; Finkelstein, A.; Ladokhin, A.S. Conformational switching of the diphtheria toxin $\mathrm{T}$ domain. J. Mol. Biol. 2010, 402, 1-7.

28. Kurnikov, I.V.; Kyrychenko, A.; Flores-Canales, J.C.; Rodnin, M.V.; Simakov, N.; Vargas-Uribe, M.; Posokhov, Y.O.; Kurnikova, M.; Ladokhin, A.S. pH-Triggered conformational switching of the diphtheria toxin t-domain: The roles of $N$-terminal histidines. J. Mol. Biol. 2013, 425, 2752-2764.

29. Vargas-Uribe, M.; Rodnin, M.V.; Kienker, P.; Finkelstein, A.; Ladokhin, A.S. Crucial role of H322 in folding of the diphtheria toxin T-domain into the open-channel state. Biochemistry 2013, 52, 3457-3463.

30. Murphy, R.F.; Powers, S.; Cantor, C.R. Endosome ph measured in single cells by dual fluorescence flow cytometry: Rapid acidification of insulin to $\mathrm{pH}$ 6. J. Cell Biol. 1984, 98, $1757-1762$.

31. Yamashiro, D.J.; Maxfield, F.R. Kinetics of endosome acidification in mutant and wild-type chinese hamster ovary cells. J. Cell Biol. 1987, 105, 2713-2721.

32. Huotari, J.; Helenius, A. Endosome maturation. EMBO J. 2011, 30, 3481-3500.

33. Shaw, D.E.; Bowers, K.J.; Chow, E.; Eastwood, M.P.; Ierardi, D.J.; Klepeis, J.L.; Kuskin, J.S.; Larson, R.H.; Lindorff-Larsen, K.; Maragakis, P.; et al. Millisecond-Scale Molecular Dynamics Simulations on Anton. In Proceedings of the Conference on High Performance Computing Networking, Storage and Analysis-SC'09, Portland, OR, USA, 14-20 November 2009; ACM Press: New York, NY, USA, 2009; p. 1. 
34. Shaw, D.E.; Chao, J.C.; Eastwood, M.P.; Gagliardo, J.; Grossman, J.P.; Ho, C.R.; Ierardi, D.J.; Kolossváry, I.; Klepeis, J.L.; Layman, T.; et al. ANTON, a special-purpose machine for molecular dynamics simulation. ACM SIGARCH Comput. Archit. News 2007, 35, 1-12.

35. Ladokhin, A.S.; White, S.H. Interfacial folding and membrane insertion of a designed helical peptide. Biochemistry 2004, 43, 5782-5791.

36. Ladokhin, A.S.; White, S.H. Protein chemistry at membrane interfaces: Non-additivity of electrostatic and hydrophobic interactions. J. Mol. Biol. 2001, 309, 543-552.

37. Posokhov, Y.O.; Gottlieb, P.A.; Morales, M.J.; Sachs, F.; Ladokhin, A.S. Is lipid bilayer binding a common property of inhibitor cysteine knot ion-channel blockers? Biophys. J. 2007, 93, L20-L22.

38. Ladokhin, A.S.; White, S.H. Folding of amphipathic $\alpha$-helices on membranes: Energetics of helix formation by melittin. J. Mol. Biol. 1999, 285, 1363-1369.

39. Fernandez-Vidal, M.; Jayasinghe, S.; Ladokhin, A.S.; White, S.H. Folding amphipathic helices into membranes: Amphiphilicity trumps hydrophobicity. J. Mol. Biol. 2007, 370, 459-470.

40. Ladokhin, A.S.; Isas, J.M.; Haigler, H.T.; White, S.H. Determining the membrane topology of proteins: Insertion pathway of a transmembrane helix of annexin 12. Biochemistry 2002, 41, 13617-13626.

41. Posokhov, Y.O.; Rodnin, M.V.; Lu, L.; Ladokhin, A.S. Membrane insertion pathway of annexin b12: Thermodynamic and kinetic characterization by fluorescence correlation spectroscopy and fluorescence quenching. Biochemistry 2008, 47, 5078-5087.

42. Rodnin, M.V.; Kyrychenko, A.; Kienker, P.; Sharma, O.; Vargas-Uribe, M.; Collier, R.J.; Finkelstein, A.; Ladokhin, A.S. Replacement of $C$-terminal histidines uncouples membrane insertion and translocation in diphtheria toxin T-domain. Biophys. J. 2011, 101, L41-L43.

43. Rodnin, M.V.; Posokhov, Y.O.; Contino-Pepin, C.; Brettmann, J.; Kyrychenko, A.; Palchevskyy, S.S.; Pucci, B.; Ladokhin, A.S. Interactions of fluorinated surfactants with diphtheria toxin T-domain: Testing new media for studies of membrane proteins. Biophys. J. 2008, 94, 4348-4357.

44. Posokhov, Y.O.; Ladokhin, A.S. Lifetime fluorescence method for determining membrane topology of proteins. Anal. Biochem. 2006, 348, 87-93.

45. Ladokhin, A.S.; Jayasinghe, S.; White, S.H. How to measure and analyze tryptophan fluorescence in membranes properly, and why bother? Anal. Biochem. 2000, 285, 235-245.

46. Chenal, A.; Prongidi-Fix, L.; Perier, A.; Aisenbrey, C.; Vernier, G.; Lambotte, S.; Haertlein, M.; Dauvergne, M.T.; Fragneto, G.; Bechinger, B.; et al. Deciphering membrane insertion of the diphtheria toxin $\mathrm{T}$ domain by specular neutron reflectometry and solid-state nmr spectroscopy. J. Mol. Biol. 2009, 391, 872-883.

47. Wimalasena, D.S.; Cramer, J.C.; Janowiak, B.E.; Juris, S.J.; Melnyk, R.A.; Anderson, D.E.; Kirk, K.L.; Collier, R.J.; Bann, J.G. Effect of 2-fluorohistidine labeling of the anthrax protective antigen on stability, pore formation, and translocation. Biochemistry 2007, 46, 14928-14936.

48. Buckley, J.T.; Wilmsen, H.U.; Lesieur, C.; Schulze, A.; Pattus, F.; Parker, M.W.; van der Goot, F.G. Protonation of histidine-132 promotes oligomerization of the channel-forming toxin aerolysin. Biochemistry 1995, 34, 16450-16455.

49. Wimley, W.C.; White, S.H. Experimentally determined hydrophobicity scale for proteins at membrane interfaces. Nat. Struct. Biol. 1996, 3, 842-848. 
50. Ren, J.; Kachel, K.; Kim, H.; Malenbaum, S.E.; Collier, R.J.; London, E. Interaction of diphtheria toxin $\mathrm{t}$ domain with molten globule-like proteins and its implications for translocation. Science 1999, 284, 955-957.

51. Kakimoto, S.; Hamada, T.; Komatsu, Y.; Takagi, M.; Tanabe, T.; Azuma, H.; Shinkai, S.; Nagasaki, T. The conjugation of diphtheria toxin $\mathrm{T}$ domain to poly(ethylenimine) based vectors for enhanced endosomal escape during gene transfection. Biomaterials 2009, 30, 402-408.

52. Zhang, Y.; Schulte, W.; Pink, D.; Phipps, K.; Zijlstra, A.; Lewis, J.D.; Waisman, D.M. Sensitivity of cancer cells to truncated diphtheria toxin. PLoS One 2010, 5, e10498.

53. Wild, R.; Yokoyama, Y.; Dings, R.P.; Ramakrishnan, S. Vegf-dt385 toxin conjugate inhibits mammary adenocarcinoma development in a transgenic mouse model of spontaneous tumorigenesis. Breast Cancer Res. Treat. 2004, 85, 161-171.

54. Urieto, J.O.; Liu, T.; Black, J.H.; Cohen, K.A.; Hall, P.D.; Willingham, M.C.; Pennell, L.K.; Hogge, D.E.; Kreitman, R.J.; Frankel, A.E. Expression and purification of the recombinant diphtheria fusion toxin dt388il3 for phase I clinical trials. Protein Expr. Purif. 2004, 33, 123-133.

55. Turturro, F. Denileukin diftitox: A biotherapeutic paradigm shift in the treatment of lymphoid-derived disorders. Expert Rev. Anticancer Ther. 2007, 7, 11-17.

56. Ramakrishnan, S.; Olson, T.A.; Bautch, V.L.; Mohanraj, D. Vascular endothelial growth factor-toxin conjugate specifically inhibits $\mathrm{kdr} / \mathrm{flk}$-1-positive endothelial cell proliferation in vitro and angiogenesis in vivo. Cancer Res. 1996, 56, 1324-1330.

57. Ramage, J.G.; Vallera, D.A.; Black, J.H.; Aplan, P.D.; Kees, U.R.; Frankel, A.E. The diphtheria toxin/urokinase fusion protein (dtat) is selectively toxic to cd87 expressing leukemic cells. Leuk. Res. 2003, 27, 79-84.

58. Murphy, J.R.; Bishai, W.; Borowski, M.; Miyanohara, A.; Boyd, J.; Nagle, S. Genetic construction, expression, and melanoma-selective cytotoxicity of a diphtheria toxin-related alpha-melanocytestimulating hormone fusion protein. Proc. Natl. Acad. Sci. USA 1986, 83, 8258-8262.

59. Kreitman, R.J. Immunotoxins for targeted cancer therapy. Aaps J. 2006, 8, E532-E551.

60. Hogge, D.E.; Yalcintepe, L.; Wong, S.H.; Gerhard, B.; Frankel, A.E. Variant diphtheria toxin-interleukin-3 fusion proteins with increased receptor affinity have enhanced cytotoxicity against acute myeloid leukemia progenitors. Clin. Cancer Res. 2006, 12, 1284-1291.

61. Hall, P.D.; Willingham, M.C.; Kreitman, R.J.; Frankel, A.E. Dt388-gm-csf, a novel fusion toxin consisting of a truncated diphtheria toxin fused to human granulocyte-macrophage colony-stimulating factor, prolongs host survival in a scid mouse model of acute myeloid leukemia. Leukemia 1999, 13, 629-633.

62. Feuring-Buske, M.; Frankel, A.; Gerhard, B.; Hogge, D. Variable cytotoxicity of diphtheria toxin 388-granulocyte-macrophage colony-stimulating factor fusion protein for acute myelogenous leukemia stem cells. Exp. Hematol. 2000, 28, 1390-1400.

63. Duvic, M.; Talpur, R. Optimizing denileukin diftitox (ontak) therapy. Future Oncol. 2008, 4, 457-469.

64. Cohen, K.A.; Liu, T.F.; Cline, J.M.; Wagner, J.D.; Hall, P.D.; Frankel, A.E. Toxicology and pharmacokinetics of $\mathrm{dt} 388 \mathrm{il} 3$, a fusion toxin consisting of a truncated diphtheria toxin (dt388) linked to human interleukin 3 (i13), in cynomolgus monkeys. Leuk. Lymphoma 2004, 45, 1647-1656. 
65. Black, J.H.; McCubrey, J.A.; Willingham, M.C.; Ramage, J.; Hogge, D.E.; Frankel, A.E. Diphtheria toxin-interleukin-3 fusion protein ( $\mathrm{dt}(388)$ il3) prolongs disease-free survival of leukemic immunocompromised mice. Leukemia 2003, 17, 155-159.

66. Reshetnyak, Y.K.; Andreev, O.A.; Lehnert, U.; Engelman, D.M. Translocation of molecules into cells by $\mathrm{pH}$-dependent insertion of a transmembrane helix. Proc. Natl. Acad. Sci. USA 2006, 103, 6460-6465.

67. Reshetnyak, Y.K.; Segala, M.; Andreev, O.A.; Engelman, D.M. A monomeric membrane peptide that lives in three worlds: In solution, attached to, and inserted across lipid bilayers. Biophys. J. 2007, 93, 2363-2372.

68. Antignani, A.; Youle, R.J. How do Bax and Bak lead to permeabilization of the outer mitochondrial membrane? Curr. Opin. Cell Biol. 2006, 18, 685-689.

69. Youle, R.J.; Strasser, A. The Bcl-2 protein family: Opposing activities that mediate cell death. Nat. Rev. Mol. Cell Biol. 2008, 9, 47-59.

70. Minn, A.J.; Velez, P.; Schendel, S.L.; Liang, H.; Muchmore, S.W.; Fesik, S.W.; Fill, M.; Thompson, C.B. Bcl-X(L) forms an ion channel in synthetic lipid membranes. Nature 1997, 385, 353-357.

71. Hsu, Y.T.; Wolter, K.G.; Youle, R.J. Cytosol-to-membrane redistribution of bax and Bcl-X(L) during apoptosis. Proc. Natl. Acad. Sci. USA 1997, 94, 3668-3672.

(C) 2013 by the authors; licensee MDPI, Basel, Switzerland. This article is an open access article distributed under the terms and conditions of the Creative Commons Attribution license (http://creativecommons.org/licenses/by/3.0/). 